\title{
Integrated maneuver and control design for ROV operations
}

\author{
Rui M. F. Gomes, João B. Sousa and Fernando Lobo Pereira \\ Instituto de Sistemas e Robótica - Porto \\ Faculdade de Engenharia da Universidade do Porto \\ Rua Dr. Roberto Frias \\ 4200-465 Porto, Portugal \\ E-mail: \{rgomes,jtasso,flp\}@fe.up.pt
}

\begin{abstract}
The integrated maneuver and control structures for a Remotely Operated Vehicle are presented in the context of the developments of "IES - Inspection of Underwater Structures" project. The project concerns the design and implementation of an advanced low cost system for the inspection of underwater structures based on a Remotely Operated Vehicle (ROV). First, the sub-systems of the IES system are described. Second, an example of a mission is outlined. Third, the control architecture is briefly sketched and formalized. Fourth, the design of the regulation and tracking controllers for this architecture are discussed. The design uses a non-linear Dynamic Surface Controller (DSC). This controller is coupled with a trajectory generation system for optimal performance. The ROV model is differentially flat under mild assumptions. The trajectory generation system uses this property to produce optimal trajectories. Finally, simulation runs of DSC and PID are compared in light of model parameters uncertainty. Extensions of the work are discussed as conclusions.

Keywords: Multivariable control, Remotely Operated Vehicles, Underwater Inspection, Dynamic Surface Control.
\end{abstract}

\section{INTRODUCTION}

The IES - Inspection of Underwater Structures project concerns the design and implementation of an advanced low cost system for the inspection of underwater structures based on a Remotely Operated Vehicle (ROV). Example missions include the evaluation of the state of corrosion of submerged steel plates and of the conservation state of underwater structures, and also archeology. The project started in 1999, has a total duration of 3 years, and is funded by PROGRAMA PRAXIS XXI - MEDIDA 3.1B, Portugal. IES is a collaborative project involving the Associação dos Portos de Douro e Leixões (APDL) and the Underwater Systems and Technology Laboratory (USTL) from Porto University. The laboratory was founded in 1997 to promote research, development, deployment, and operation of advanced systems and technologies in oceanographic and environment field studies. Today, USTL aggregates close to 20 researchers including Faculty, Ph.D. and M.Sc. students, and engineers. The USTL started developing and operating the Isurus Autonomous Underwater Vechicle in 1997. Since then, USTL designed and developed: 1) Remotely Operated Vehicle (ROV) for the inspection of underwater structures; 2) low cost AUV for coastal oceanography [3]; 3) low cost sensor modules for remote environmental data collection; 4) acoustic navigation technology for multiple AUVs [11]; and 5) feasible concepts for the networked operation of multiple vehicles and systems [7]. Over the last two years the USTL devoted an intense effort to the development of feasible concepts for the networked operation of multiple vehicles and systems, and the first deployments are scheduled for late 2003.

This paper presents the integrated maneuver and control structures for the IES project and is organized as follows. In section II, we describe the main sub-systems of the IES system. Section III presents an example of a typical mission. Section IV gives a short overview of the control architecture implemented in the ROV. Section V addresses the control system design and presents simulations results. Finally, section VI ends with some concluding remarks, and future work.

\section{SYSTEMS}

Except for the ROV frame, hull and thrusters, all of the other components and systems were designed and implemented at USTL. The ROV frame, hull and thrusters are a customized version of the Deep Ocean $500 \mathrm{~S}$ model from Deep Ocean Engineering. The main difference with respect to the standard model is an additional cylinder that houses electronics and sensors.

The IES system integrates the following innovative technologies and systems developed at the Underwater Systems and Technology Laboratory (LSTS) from Porto University:

- Acoustic navigation system [11], [2].

- Advanced control systems.

- Power and motor control.

In the basic configuration the IES system comprises the following systems:

Computer system - Consists of a PC-104 stack running the real-time QNX operating system on the vehicle, and a Windows based PC connected through an Ethernet cable. The PC-104 stack is housed in the main cylinder of the ROV, and controls the ROV hardware through a CAN bus (fig 2 on the right). Some systems also have an RS-232 interface, and therefore a PC-104 RS-232 board was added on the stack. Additional sensors are interfaced through an A/D card on the PC-104 bus. The Windows based PC runs the operator console. The PC also runs a Web server providing Web-based access to obtain data from operations, while ROV control is restricted to the operator console. The PC-104 computer system runs the command, control and navigation software. Basically, this 

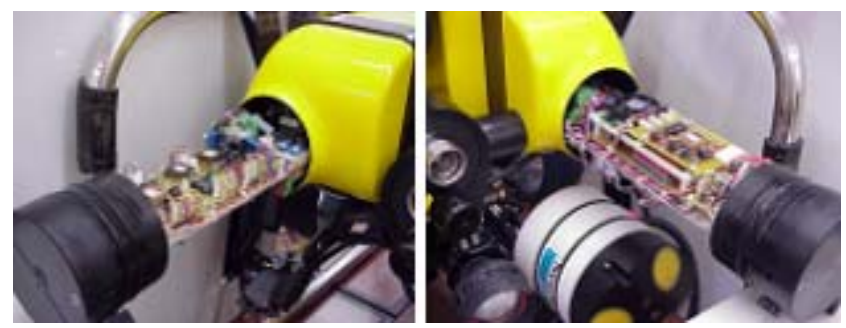

Fig. 1. On the left the starboard cylinder with an electronic compass and the dimmer and motor controllers. On the right the port cylinder with the on-board power supply system and also it's monitor.
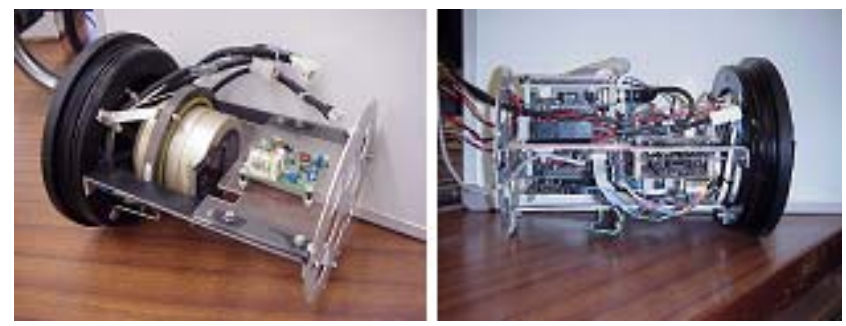

Fig. 2. On the left the inertial system and on the right the PC104 CPU stack

computer accepts high-level commands from the console, and informs the console about the system state.

Power system - A portable generator provides electrical power to the system. The umbilical cable feeds the ROV with two main power lines $120 \mathrm{~V}$ DC / 1,2KW and 48V / 100W. The first power line feeds DC motors and lights. The other line feeds the on-board electronics. Inside the ROV, there is a power conversion unit (fig 1 on the right) to generates all of the required voltage levels. Those levels are achieved with dc-dc converters. This arrangement of the power system minimizes the number of wires in the umbilical cable and consequently its weight. This design option is aimed to minimize the effects of the tether on the ROV dynamics, one of the traditional difficulties associated with ROV operations.

Motor control system - This system comprises two CAN nodes housed in the two upper vehicle cylinders. The controllers generate the reference PWM signals to the four thruster power drives. The DC motors are powered by these drives.

Navigation system - The suite of navigation sensors includes the on-board sensors and external ones [11], [2]. The available sensors are: magnetic compass, inclinometers, inertial navigation unit, depth cell, altimeter, Doppler Velocity Log (DVL) and LBL acoustic positioning system.

The acoustic system uses two acoustic beacons. In normal operation, the ROV system sends an acoustic signal to each beacon and waits for their response. The time that takes from the transmission to the reception gives the distance between the ROV and each of the acoustic beacons. The DVL sensor gives the velocity of the vehicle relative to water or relative to ground. The inertial navigation unit is also housed in the ROV (fig 2 on the left). The vehicle's depth is measured by the pressure sensor. The compass (fig 1 on the left)

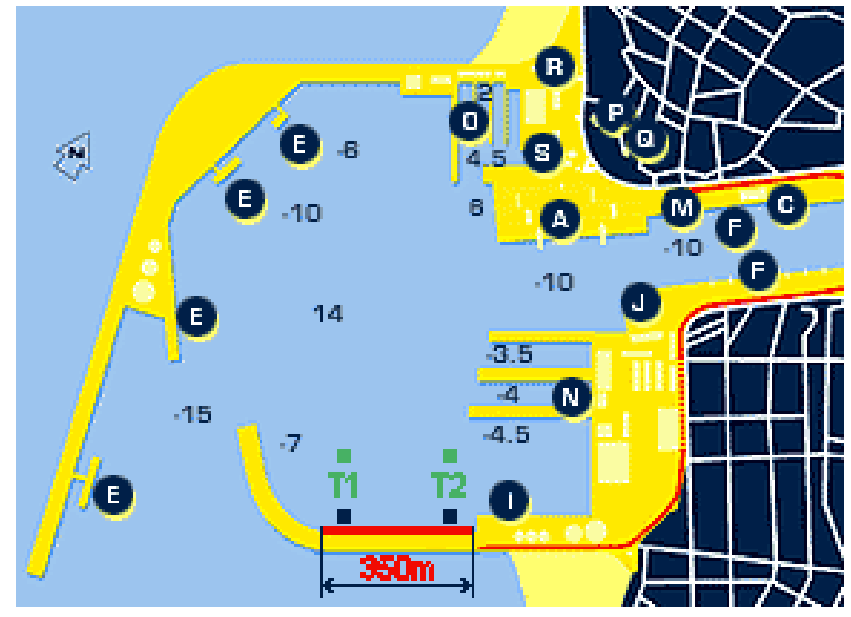

Fig. 3. APDL harbour map

gives measurements of the orientation of the vehicle. It also measures the roll and pitch angles. We use an altimeter to measure the distance of the ROV from vertical walls.

We developed a sensor fusion algorithm to estimate all of the state variables, which are three positions, three orientations, and six velocities.

Vision system - In the basic configuration, the vision system consists of a camera mounted in a pan-and-tilt unit and a spot light. The video image is converted to the digital format in the on-board frame-grabber, and sent to the operator console through the Ethernet connection.

The basic inspection configuration can be enhanced with a set of plug-and-play inspection and intervention tools. This set comprises another vision system and an array of magnetic sensors. Other tools that are being considered for development include a tactile sensor array, and a scraping device.

\section{MISSION EXAMPLE: APDL 18-10-2002}

The Port of Leixões comprises the largest seaport infrastructure in the north of Portugal and is one of the most important seaports in the country. With $5 \mathrm{~km}$ of quays, 55ha of embankments and 120ha of wet area, Leixões has excellent road, rail and maritime accesses and is equipped with advanced information systems for vessels traffic control and management.

Representing $25 \%$ of the Portuguese foreign trade (about 10 Million Euros of goods) and handling 14 million tons of commodities a year, the Port of Leixões is one of the most competitive and versatile multi-purpose ports in the country. 3,100 vessels a year come through Leixões, carrying all sorts of goods: textiles, granites, wines, timber, vehicles, cereals, containers, scrap metal, iron and steel, alcohol, schnapps, sugar, oil, molasses, petroleum products, and even passengers from Cruise Liners [1].

In what concerns the visual inspection of underwater infrastructures for maintenance the challenges are:

- The state of corrosion of submerged steel pillars. 


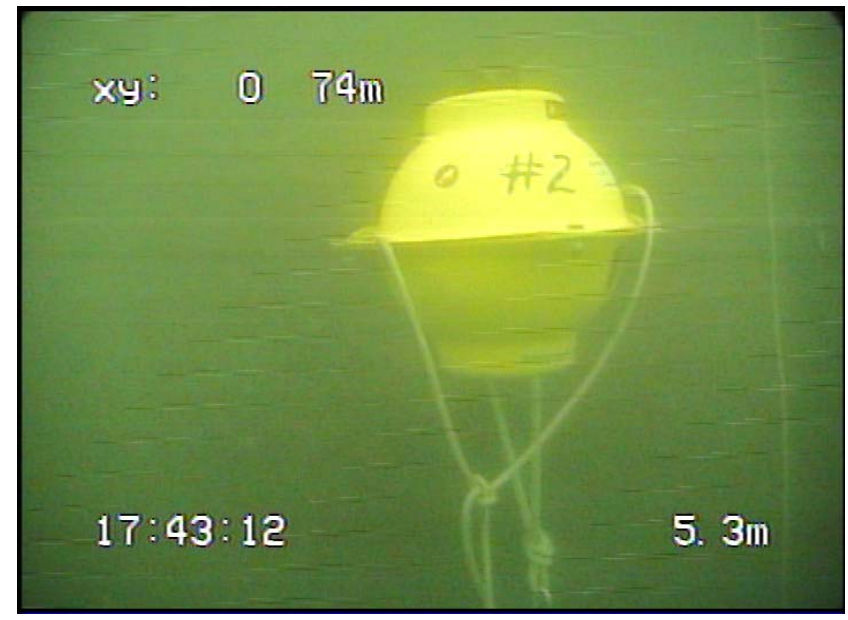

Fig. 4. Picture of one of our transponders

- The state of corrosion of all kinds of vessels.

- The state of the quay stone walls. The walls are subject to high pressures due to the motions of some types of thrusters.

In what follows we describe a typical inspection mission. The objective of this mission is to check the state of the quay wall marked with a solid red line close to the I letter in figure 3. The quay is 350 meters long and the average depth is 7 meters which leads to an area of inspection of almost $2500 \mathrm{~m}^{2}$. This wall is made of stone blocks. The visual inspection consists in determining if all of the stone blocks are correctly positioned.

\section{A. Mission setup}

There are two ways to operate the ROV at the APDL facilities: from a small boat or from the quay. We choose to operate from the quay.

For this mission there are two possible ways to operate the vehicle. One of them is to install the transponders in a way that we get absolute positioning measures on both $\mathrm{x}$ and $\mathrm{y}$ DOFs (Degree Of Freedom) horizontal plane. The other is to get measures on only one degree of freedom ( $\mathrm{x}$ or $\mathrm{y}$ ). The difference between both approaches is on the way that the transponders are installed.

Traditionally we moor the two transponders (T1, T2) away from the wall in order to measure the $\mathrm{x}$ and $\mathrm{y}$ position of the ROV (see on picture 3 the green squares). However, in this mission there was no need for absolute position measuring on both DOFs. Therefore we decided to deploy the transponders close to the wall (see on picture 3 the dark blue squares) to operate on the base line. The picture 4 shows one of the transponders. This way we are able to get a better accuracy in that degree of freedom.

After installing the transponders we measure their positions and load this data into the vehicle navigation software.

\section{B. Mission execution}

There are two modes of operation: tele-operation and teleprograming. In the first one the operator has the ability to pilot the vehicle with a joystick. In the second one the operator fills in the parameters of a template maneuver and commands its autonomous execution.

When we started the inspection we realized that the visibility was quite poor due to pollution. This lack of visibility forced the operator to reduce the distance between the vehicle and the wall. Some images captured by the ROV during the inspection process are presented in figures 5 and 6 . These pictures also show the amount of marine growth in all of these stones. Notice that the display superimposes the vehicle position and current time on the image. In figure 5 a) we can observe a fissure between two consecutive blocks. In the figures $5 \mathrm{~b}$ ) and 6 we realize that the darker part corresponds to missing blocks.

Including preparation and setup this mission took less than two days. One of the innovative aspects of the operation of the IES system is that it allows for the specialists to actually pilot the ROV. This allows them to study in detail and in real-time all of the features of the images taken by the ROV.

\section{Control Architecture}

\section{A. Maneuvers}

We organize the operations of the ROV in terms of prototypical maneuvers. In practical terms a maneuver encapsulates a pattern of interactions with: 1) low-level control and navigation systems; 2) trajectory generation modules; 3) sensors; and 4) operator.

We have defined a set of 4 atomic maneuvers from which all of the ROV maneuvers can be derived: teleoperation, followtrajectory, followpath, hover. These atomic maneuvers are parameterized to accommodate the user specifications. For example the parameters for the followtrajectory maneuver include: trajectory, distance from a wall, initial and final points.

We specify a maneuver as an open hybrid automaton 1. Each atomic maneuver is modelled by a 4 state hybrid automaton. The states are initial, normal, error, done. The maneuver logic is encoded as transitions among these states and invariants for each state: 1) the conditions under which the maneuver are valid are modelled as invariants for the initial, normal, done states; the conditions under which the maneuver can start are specified as the guard for the transition from initial to normal; the conditions under which the maneuver is terminated successfully are specified as the guard from normal to done; etc.. To each state we associate a low-level controller, and a trajectory or path generation module.

${ }^{1}$ A hybrid automaton consists of control locations with edges between the control locations. The control locations are the vertices in a graph. A location is labelled with a differential inclusion, and every edge is labelled with a guard, and a jump and reset relation. Formally, a hybrid automaton is $\mathrm{H}=$ (L,D,E) where:

- L is a set of control locations.

- $\mathrm{D}: \mathrm{L} \rightarrow$ Inclusions where $\mathrm{D}(\mathrm{l})$ is the differential inclusion at location 1 .

- $\mathrm{E} \subseteq L X$ Guard $X$ Jump $X L$ are the edges - an edge e $=(1, \mathrm{~g}, \mathrm{j}, \mathrm{m}) \epsilon \mathrm{E}$ is an edge from location from 1 to $\mathrm{m}$ with guard $\mathrm{g}$ and jump relation $\mathrm{j}$.

The state of a hybrid automaton is a pair $(l, x)$ where 1 is the control location and $x \in R^{n}$ is the continuous state. 

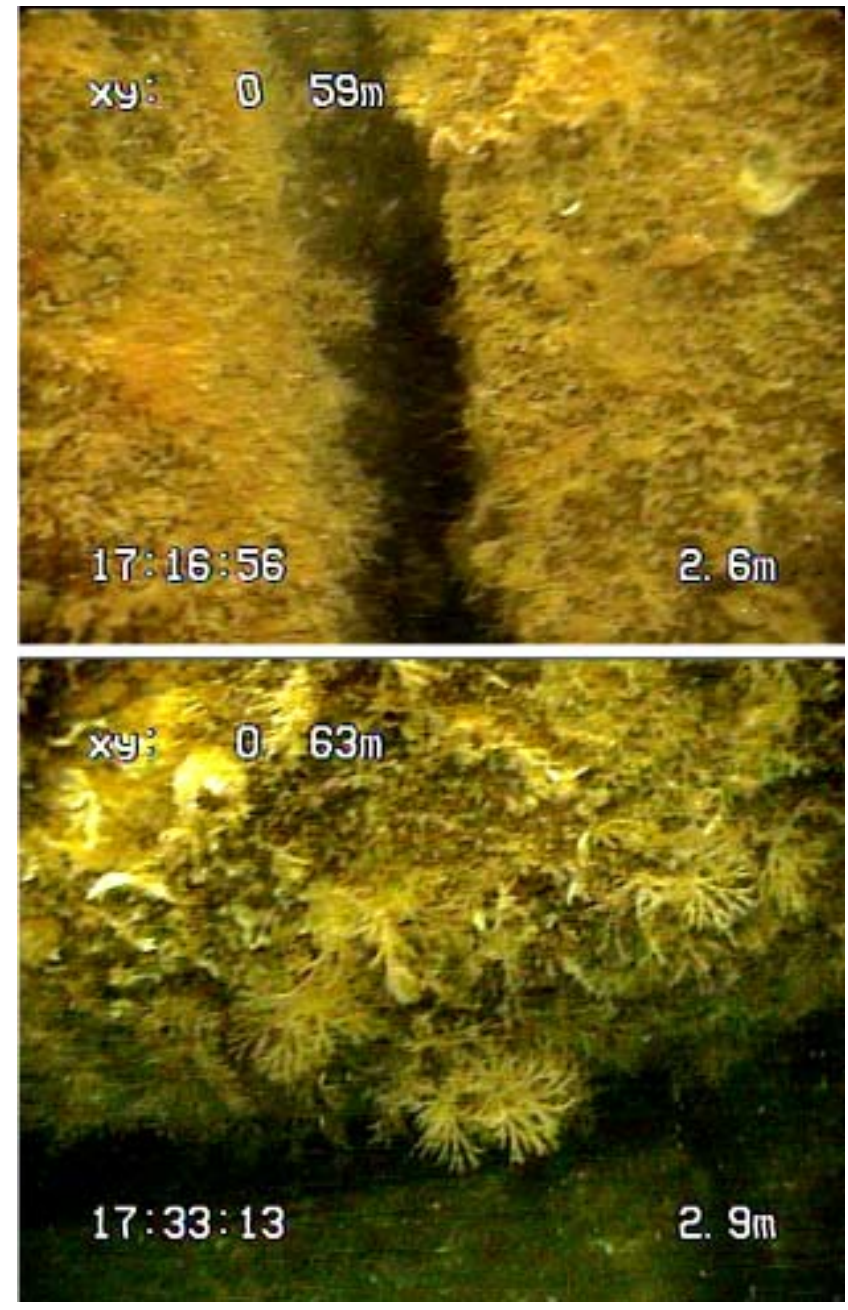

Fig. 5. Images captured by the vehicle. a) The upper image displays fissures between two consecutive stone blocks. b) The lower image shows the absence of at least one stone block.

We build complex maneuvers from the atomic ones, where each state of a complex maneuver corresponds to the execution of an atomic one.

\section{B. Components}

The modular design of maneuvers is mirrored by the modular design of the control architecture.

The main components in our modular design, starting at the bottom of the control architecture, are: Vehicle controller - controls the execution of a maneuver (there is one controller per vehicle maneuver); Vehicle supervisor - it does not change throughout the life span of the vehicle, interfaces each vehicle with external control structures, and supervises the execution of vehicle maneuvers; Plan supervisor - supervises the execution of the mission plan thus commanding maneuvers to be executed; Mission plan - a data structure defining a partial order on tasks or maneuvers to be executed by the vehicle.
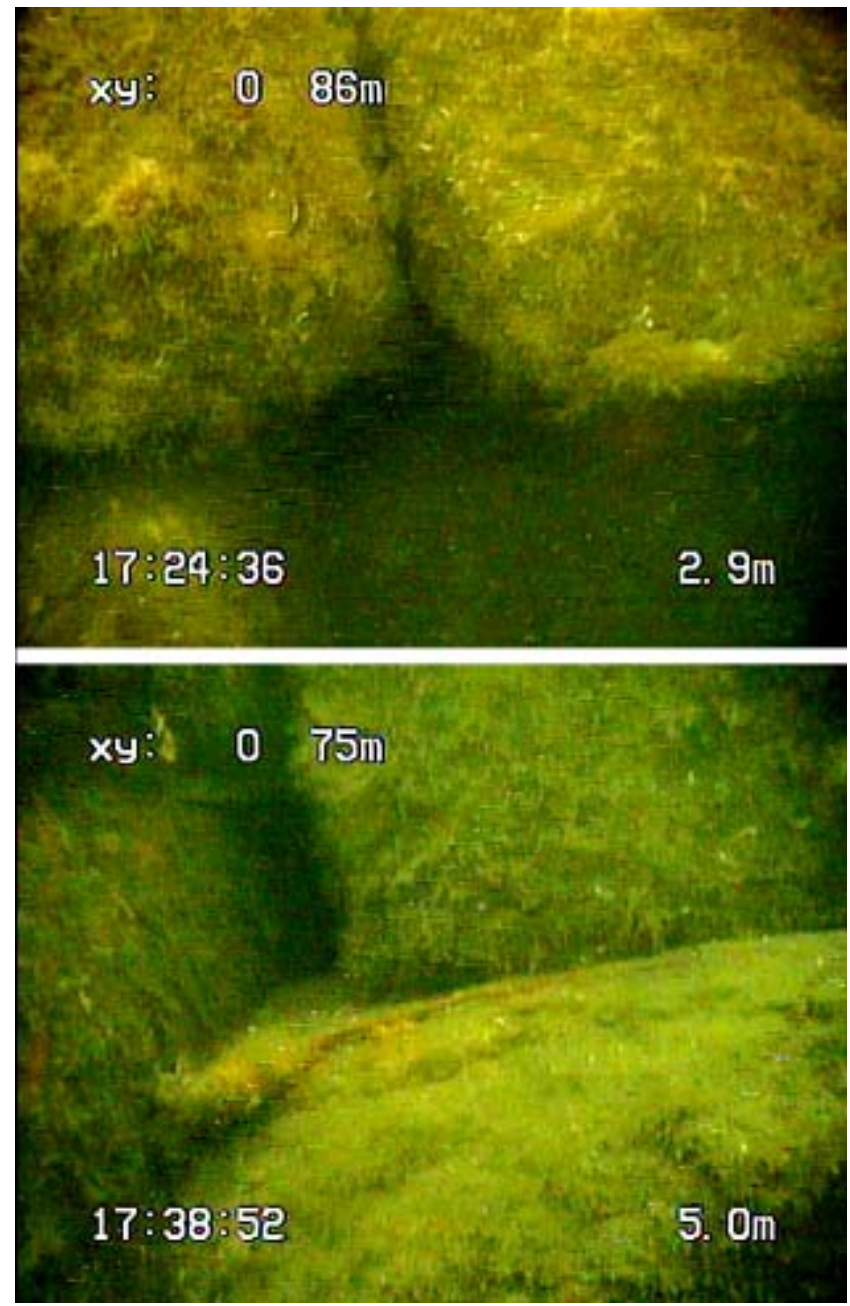

Fig. 6. Images captured by the vehicle. In both images we detect the absence of at least one stone block.

\section{LOW LEVEL CONTROL DESIGN}

In this section, we use a Dynamic Surface Controller DSC to deal with the ROV regulation problems. This controller is an improvement of the Multiple Sliding Surface controller (MSS). The MSS controller is based on backstepping and sliding modes techniques and therefore suffers from the explosion of terms in the control law (refer to example shown in [13]). DSC was developed to overcome this problem through the use of dynamic filters. With this methodology the robustness of the controller without a large number of terms is guaranteed. Moreover, the MSS controller requires finding the derivatives of the reference trajectories which is not very good from the implementation point of view.

The ROV equations of motion are described first.

\section{A. ROV Model}

The ROV model is composed of three main components: the rigid body, the propeller and the DC motor [5], [10], [12].

The rigid body model is derived from the Newton-Euler formulation. The Newton-Euler formulation is based on New- 
ton's Second Law and concerns the conservation of both linear and angular momentum.

It is important to consider two coordinate frames: the bodyfixed and the earth-fixed. The body-fixed is attached to the vehicle. Its origin is normally fixed on the center of gravity. The motion of the body-fixed reference frame is described in relation to the earth-fixed reference frame. The earth-fixed reference frame can be considered inertial for low velocity vehicles such as ROVs. The notation defined by SNAME (Society of Naval Architects and Marine Engineers) is as follows:

Position and orientation (earth-fixed):

$$
\eta=\left(\eta_{1}, \eta_{2}\right)=(x, y, z, \phi, \theta, \psi)
$$

Linear and angular velocity (body-fixed):

$$
v=\left(\nu_{1}, \nu_{2}\right)=(u, v, w, p, q, r)
$$

The velocities in both reference frames are related through the following transformation which is based on the Euler angles

$$
\dot{\eta}=J\left(\eta_{2}\right) v
$$

This transformation is undefined for $\theta= \pm 90^{\circ}$. To overcome this singularity, a quaternion approach must be considered. This is not a problem for the IES project since the vehicle does not operate close to $\theta= \pm 90^{\circ}$. Moreover, the vehicle is stable in roll and pitch, and the thruster actuation is not enough to force the vehicle to operate close to those angles.

In the body-fixed frame the nonlinear equations of motion are:

$$
\begin{gathered}
M \dot{v}+C(v) v+D(v) v+g(\eta)=\tau \\
\dot{\eta}=J\left(\eta_{2}\right) v
\end{gathered}
$$

where:

$v \quad$ Velocity of the vehicle in the body-fixed frame

$\eta \quad$ Position and the orientation of the vehicle in the inertial frame

$M \quad$ Inertia and added mass matrix of the vehicle

$C(n) \quad$ Coriolis and centripetal matrix

$D(n) \quad$ Damping matrix

$g(\eta) \quad$ Restoring forces and moments

$\tau \quad$ Body-fixed forces from the actuators and other disturbances as currents and waves. [8].

More details about all this matrices, can be found in [4],

Each thruster is composed of a motor (in our case a DC motor), a propeller, and a propeller shroud [5], [9]. Traditionally, the propeller model is a bit difficult to achieve. Some of the difficulties are:

- the separation of the drag forces of the vehicle and the propeller thrust

- complex hydrodynamic behaviors like vortex shedding on the propeller blades, un-modelled blades, duct effects

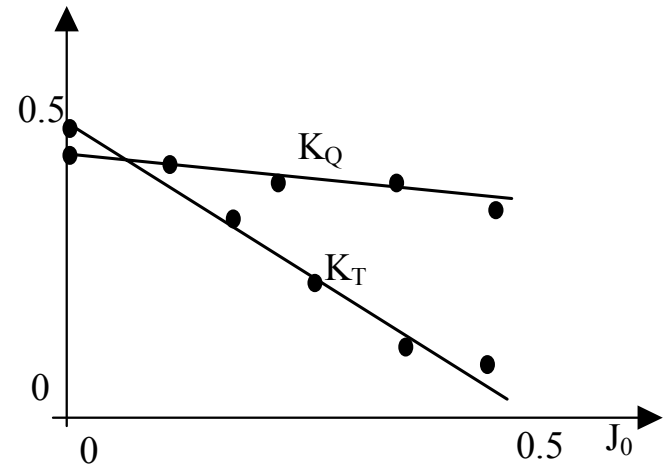

Fig. 7. Propeller parameters

The thrust force produced by the propeller depends both on the vehicle and the propeller velocities. We consider the following model.

$$
T=\rho D^{4} K_{T}\left(J_{0}\right)|n| n
$$

where

$\rho \quad$ Water density

$D \quad$ Propeller diameter

$n \quad$ Propeller revolution (rps)

$K_{T}\left(J_{0}\right) \quad$ Propeller coefficient

$J_{0} \quad$ Advance number

The advance number is given by

$$
J_{0}=\frac{V_{A}}{n D}
$$

where $V_{A}$ is the advance speed.

The advance speed is the speed of the water going through the propeller.

The data for the model is obtained experimentally. The objective is to collect several $\left(J_{0}, T\right)$ pairs. This pairs are converted into $\left(J_{0}, K_{T}\right)$ pairs with the following expression.

$$
K_{T}=\frac{T}{\rho D^{4}|n| n} .
$$

Those pairs can be represented in a graphic, like the one presented in figure 7. Once we have a large number of pairs, it is possible to get a linear regression which leads to

$$
K_{T}=\alpha_{1}+\alpha_{2} J_{0} .
$$

The final thrust force is given by

$$
T=\rho D^{4}\left(\alpha_{1}+\alpha_{2} J_{0}\right)|n| n .
$$

The motor equations are well known. There is an electric equation

$$
L_{a} \frac{d i_{a}}{d t}=-R_{a} i_{a}-K_{e} \omega+u_{a}
$$

and a mechanical one

$$
J \frac{d \omega}{d t}=K_{M} i_{a}-Q
$$

where 
$L_{a} \quad$ Armature inductance

$R_{a} \quad$ Armature resistance

$i_{a} \quad$ Armature current

$u_{a} \quad$ Armature voltage

$J \quad$ Momentum of inertia

$Q \quad$ Load torque

$K_{e} \quad$ Electric motor constant

$K_{m} \quad$ Motor torque constant

Typically the dynamics of the DC motor are neglected since it is much faster than the vehicle dynamics.

\section{B. Dynamic Surface Controller}

In this section we will deal with the problem of calculating the thruster forces to be applied to the ROV in order to reach a specific position in inertial coordinates. To do this, a Dynamic Surface controller (DSC) was addressed [6]. This controller involves two sliding surfaces. The first surface is defined as the position error

$$
S_{1}=\eta-\eta_{d}
$$

The derivative of $S_{1}$ yields

$$
\dot{S}_{1}=J(\eta) v-\dot{\eta}_{d}
$$

and the desired velocity can be defined as

$$
v_{d}=J^{-1}(\eta)\left[\dot{\eta}_{d}-\Lambda S_{1}\right]
$$

With this velocity it is guaranteed that

$$
\dot{S}_{1}+\Lambda S_{1}=0
$$

where $\Lambda$ is a positive definite matrix. To guaranty that $S_{1} \rightarrow 0$ with a proper convergence rate, a proper $\Lambda$ matrix must be designed. Moreover, if $S_{1} \rightarrow 0$ then $\eta \rightarrow \eta_{d}$.

The second sliding surface could be defined as $S_{2}=v-v_{d}$ but this approach is not very good since model differentiation is required. This requires a good model which is not always the case! In this case the DSC eliminates the need for model differentiation by passing $v_{d}$ through a bank of first order filters

$$
T \dot{\bar{v}}_{d}+\bar{v}_{d}=v_{d}
$$

$T$ is the filter time constants matrix. The time constants should be as lower as possible. From now on, $\bar{v}_{d}$ is used instead of $v_{d}$ and its derivative is easily computed as

$$
\dot{\bar{v}}_{d}=T^{-1}\left(v_{d}-\bar{v}_{d}\right) \text {. }
$$

The second sliding surface is defined as

$$
S_{2}=v-\bar{v}_{d}
$$

Now, a Lyapunov function is defined:

$$
V=\frac{1}{2} S_{2}^{T} M S_{2}
$$

Differentiating $\mathrm{V}$ and using (1) and (10) leads to

$$
\begin{aligned}
\dot{V} & =S_{2}^{T}\left[M \dot{v}-M \dot{\bar{v}}_{d}\right] \\
& =S_{2}^{T}\left[\tau-C(v) v_{d}-D(v) v_{d}-g(\eta)-M \dot{\bar{v}}_{d}\right] .
\end{aligned}
$$
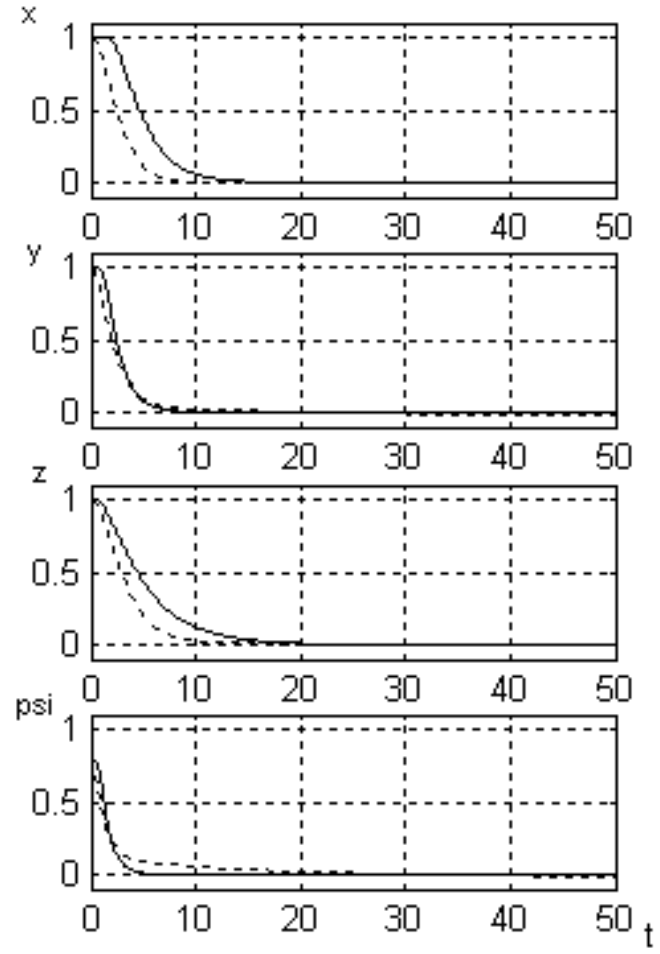

Fig. 8. Comparition between DSC and PID controllers

Taking

$$
\tau=C(v) v_{d}+D(v) v_{d}+g(\eta)+M T^{-1}\left(v_{d}-\bar{v}_{d}\right)-K_{D} S_{2}
$$

forces $\dot{V}$ to be negative.

$$
\dot{V}=-S_{2}^{T} K_{D} S_{2}
$$

Also a discontinuous term is added to the controller to achieve robustness to parameter uncertainty.

$$
\begin{aligned}
\tau= & C(v) v_{d}+D(v) v_{d}+g(\eta)+M T^{-1}\left(v_{d}-\bar{v}_{d}\right) \\
& -K_{D} S_{2}-K_{2} \operatorname{sgn}\left(S_{2}\right)
\end{aligned}
$$

The controller (12) was implemented in the simulation of the model presented in section V-A. The inputs of this controller are:

- The desired position $\eta_{d}=\left[\begin{array}{llll}x_{d} & y_{d} & z_{d} & \psi_{d}\end{array}\right]$ (inertial)

- The desired velocity $v_{d}=\left[\begin{array}{llll}u_{d} & v_{d} & w_{d} & r_{d}\end{array}\right]$ (body)

The outputs are obviously the thruster forces $\tau$ to be applied to the ROV.

Lets consider a regulation problem where the vehicle is at the inertial coordinates $(1 \mathrm{~m}, 1 \mathrm{~m}, 1 \mathrm{~m}, \pi / 4 \mathrm{rad})$ and we want to stabilize it at the origin $(0 \mathrm{~m}, 0 \mathrm{~m}, 0 \mathrm{~m}, 0 \mathrm{rad})$. Two different simulations were performed for comparative purposes. One with the DSC controller and the other with a PID controller. The results of those simulations are presented in figure 8 (DSC in solid line and PID in dashed line). The corresponding actuation is presented in figure 9. Both controller parameters were manually tuned in order to achieve the best performance. 

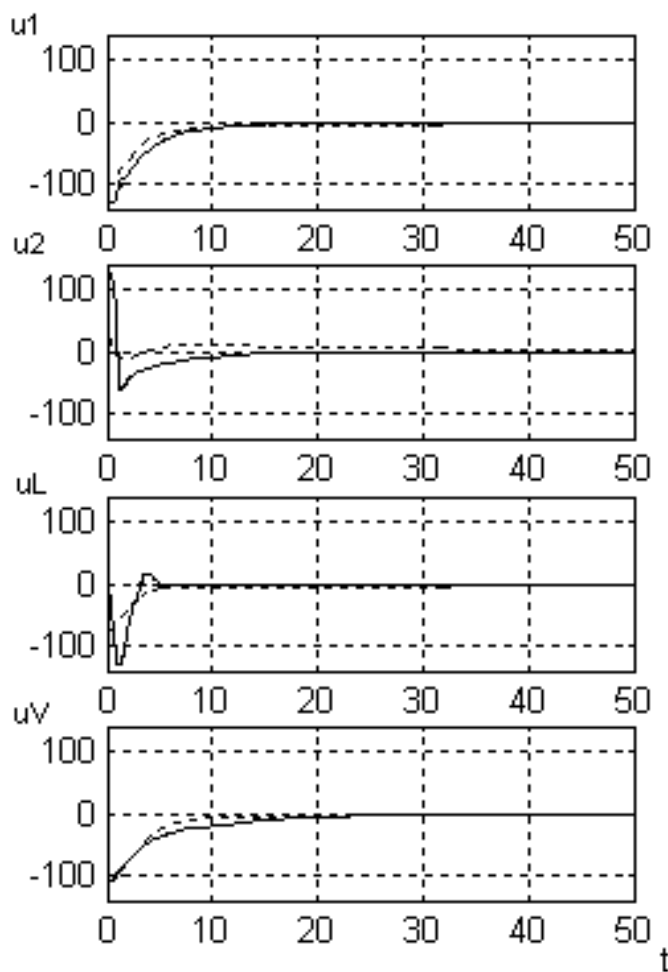

Fig. 9. Thruster actuation

In figure 8 we observe that the responses are not too different. The problem arises when there are uncertainties in the model parameters. To test the robustness of the controller to model parameters uncertainties we conducted another simulation. This time, the model parameters were modified in $50 \%$ of the nominal ones. In figure 10 we see that the PID response presents an overshoot while the DSC controller still stabilizes the vehicle perfectly. Moreover, other tests revealed that the PID controller response is seriously compromised if we change the type of disturbance.

\section{CONCLUSIONS}

This paper presented an integrated maneuver and control framework for the IES Remotely Operated Vehicle. The main concepts in this framework are maneuver, vehicle supervisor, plan supervisor, mission plan and operator. The maneuver encodes the logic required to control the vehicle to execute autonomously or assisted by the operator a complex operation. We encode the maneuver with a hybrid automaton. The vehicle supervisor supervises the execution of each maneuver and the plan supervisor supervises the execution of a mission plan. This design accommodates two modes of operation: teleoperation and tele-programming. In the first mode the vehicle supervisor supervises the execution of the tele-operation maneuver. This is a special maneuver since it basically checks for safety of the ROV while commands from the pilot. In the second mode the vehicle is controlled by all of the components of the control architecture.
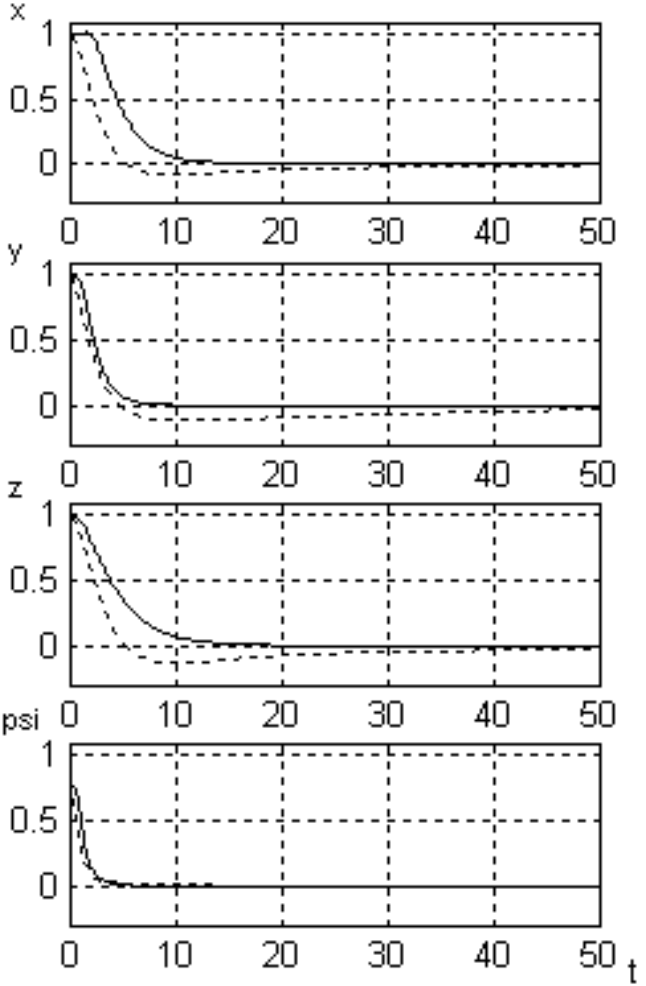

Fig. 10. System response with 50\% parameters uncertainty.

One of the main difficulties of low level control design concerns modelling and identification. The ROV model is highly non-linear, the interactions with the environment are difficult to model, and the tether is the source of a considerable disturbance. On the other hand model identification is quite complex and time consuming. In order to cope with model uncertainty we implemented and tested a robust controller - Dynamic Surface Control. We compared the performance of this controller with that of a PID controller. In the case the parameters of the model are known the performance of both controllers is not significantly different. However, the performance of the DSC controller is significantly better in the case of uncertainties in model parameters. We are planning to field test this controller in operational missions this summer, and to design an adaptive gain scheduling scheme to improve performance.

\section{ACKNOWLEDGMENTS}

This material is based upon work funded by the Programa PRAXIS XXI - Medida 3.1b) (IES project) and by Ministério da Defesa, Portugal.

\section{REFERENCES}

[1] APDL. http://www.apdl.pt.

[2] N. Cruz, L. Madureira, A. Matos, and F. Pereira. A versatile acoustic beacon for navigation and remote tracking of multiple underwater vehicles. MTS/IEEE Oceans '01, Honolulu, HI, U.S.A, 2001. 
[3] Nuno Cruz, Aníbal Matos, Alfredo Martins, Jorge Silva, Domingos Santos, Dmitri Boutov, Diogo Ferreira, and F. Lobo Pereira. Estuarine environment studies with ISURUS, a REMUS class AUV. OCEANS, 1999.

[4] Thor I. Fossen. Guidance and Control of Ocean Vehicles. John Whiley and Sons, 1995

[5] Thor Inge Fossen. Nonlinear Modelling and Control of Underwater Vehicles (PHD). 1991.

[6] Anouck R. Girard. Hybrid System Architectures for Coordinated Vehicle Control (PHD). 2002.

[7] Anouck Renée Girard, João Borges de Sousa, and J. Karl Hedrick. AN OVERVIEW OF EMERGING RESULTS IN NETWORKED MULTIVEHICLE SYSTEMS. CDC, 2001.

[8] Rui M. F. Gomes, João B. Sousa, and Fernando L. Pereira. Modeling and control of the IES project ROV. ECC 2003, Cambridge, UK.

[9] D. Yoerger L. Whitcomb. Development, comparison, and preliminary experimental validation of nonlinear dynamic thruster models. IEEE Journal of Oceanic Engineering, Vol. 24, 1999.

[10] E. Lewis. Principles of Naval Architecture. Society of NavalArchitects and Marine Engineers, 1989.

[11] A. Matos, A. Martins, N. Cruz, and F. Pereira. Development and implementation of a low-cost LBL navigation system for an AUV. MTS/IEEE Oceans '99, Seattle, U.S.A, 1999.

[12] J. N. Newman. Marine Hydrodynamics. MIT Press, 1977.

[13] D. Swaroop, J. K. Hedrick, P. P. Yip, and J. C. Gerdes. Dynamic surface control for a class of nonlinear systems. IEEE Transactions on Automatic Control, 45, 2000 